\title{
COMPARISON OF DIFFERENT NUMERICAL SCHEMES FOR THE CAHN-HILLIARD EQUATION
}

\author{
SEUNGGYU LEE ${ }^{1}$, CHAEYOUNG LEE $^{1}$, HYUN GEUN LEE ${ }^{2}$, AND JUNSEOK KIM ${ }^{1 \dagger}$ \\ ${ }^{1}$ Department of Mathematics, Korea University, South Korea \\ ${ }^{2}$ Institute of Mathematical Sciences, Ewha W. University, South Korea \\ E-mail address: cfdkim@korea.ac.kr
}

\begin{abstract}
The Cahn-Hilliard equation was proposed as a phenomenological model for describing the process of phase separation of a binary alloy. The equation has been applied to many physical applications such as a morphological instability caused by elastic non-equilibrium, image inpainting, two- and three-phase fluid flow, phase separation, flow visualization and the formation of the quantum dots. To solve the Cahn-Hillard equation, many numerical methods have been proposed such as the explicit Euler's, the implicit Euler's, the Crank-Nicolson, the semi-implicit Euler's, the linearly stabilized splitting and the non-linearly stabilized splitting schemes. In this paper, we investigate each scheme in finite-difference schemes by comparing their performances, especially stability and efficiency. Except the explicit Euler's method, we use the fast solver which is called a multigrid method. Our numerical investigation shows that the linearly stabilized stabilized splitting scheme is not unconditionally gradient stable in time unlike the known result. And the Crank-Nicolson scheme is accurate but unstable in time, whereas the non-linearly stabilized splitting scheme has advantage over other schemes on the time step restriction.
\end{abstract}

\section{INTRODUCTION}

The Cahn-Hilliard $(\mathrm{CH})$ equation was originated from a model of the phase separation which is called the spinodal decomposition in a binary alloy $[1,2]$. Since the spinodal decomposition is one of few phase transformation models in solids, the equation has been applied to various problems in theoretical and experimental materials science fields such as a morphological instability caused by elastic non-equilibrium [3], image inpainting [4], multiphase fluid flow [5, 6, 7, 8], phase separation [9], flow visualization [10] and the formation of the quantum dots [11]. The $\mathrm{CH}$ equation is as follows:

$$
\begin{aligned}
\frac{\partial c(\mathbf{x}, t)}{\partial t} & =\nabla \cdot[M(c(\mathbf{x}, t)) \nabla \mu(c(\mathbf{x}, t))], \mathbf{x} \in \Omega, 0<t \leq T, \\
\mu(c(\mathbf{x}, t)) & =F^{\prime}(c(\mathbf{x}, t))-\epsilon^{2} \Delta c(\mathbf{x}, t),
\end{aligned}
$$

Received by the editors June 8 2013; Accepted July 12013.

2000 Mathematics Subject Classification. 65M06.

Key words and phrases. Cahn-Hilliard equation, Comparison study, Finite-difference method.

${ }^{\dagger}$ Corresponding author. 
where $\Omega \subset \mathbb{R}^{d}(d=1,2,3)$ is a domain. The quantity $c(\mathbf{x}, t)$ is defined to be the difference between the concentrations of the two mixture components (e.g., $\left(m_{1}-m_{2}\right) /\left(m_{1}+m_{2}\right)$ where $m_{1}$ and $m_{2}$ are the masses of components 1 and 2 in a representative volume $V$ ) and $\mu(c(\mathbf{x}, t))$ is identified as a chemical potential. The coefficient $M(c)>0$ is a diffusional mobility depending on $c . F(c)=0.25\left(1-c^{2}\right)^{2}$ is the Helmholtz free energy which has a double well potential (see Fig.1) and the small constant $\epsilon$ is the gradient energy coefficient related to the interfacial energy. The $\mathrm{CH}$ equation satisfies the zero Neumann condition or called the no flux boundary condition which is given as below:

$$
\frac{\partial c}{\partial n}=\frac{\partial \mu}{\partial n}=0 \text { on } \partial \Omega,
$$

where $n$ is the unit normal vector to a boundary of the domain $\partial \Omega$, so $\frac{\partial}{\partial n}$ denotes the normal derivative on $\partial \Omega$. The $\mathrm{CH}$ equation arises from the Ginzburg-Landau free energy functional

$$
\mathcal{E}(c):=\int_{\Omega}\left(F(c)+\frac{\epsilon^{2}}{2}|\nabla c|^{2}\right) d \mathbf{x} .
$$

If we differentiate the energy functional $\mathcal{E}$ and the total mass $\int_{\Omega} c d \mathbf{x}$ of mixture components with respect to time $t$ and apply the no flux boundary condition (1.3), then we get the total energy non-increasing and the total mass conserving properties, i.e.,

$$
\frac{d}{d t} \mathcal{E}(t) \leq 0 \text { and } \frac{d}{d t} \int_{\Omega} c d \mathbf{x}=0,
$$

respectively. And further detail derivation of above results is presented in [12].

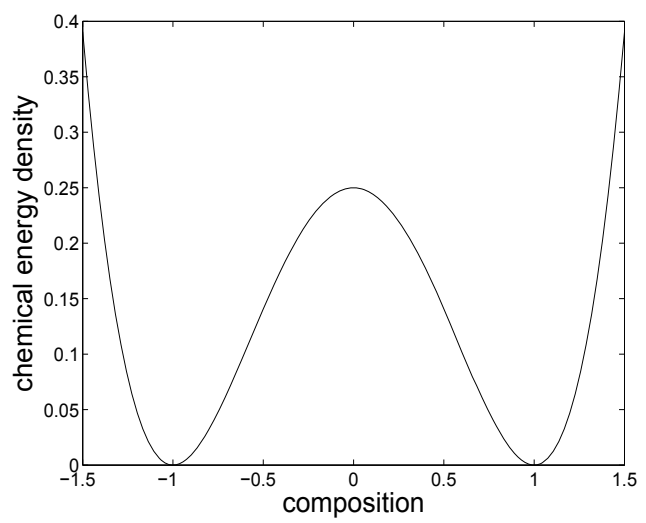

FigURE 1. A double well potential $F(c)=0.25\left(1-c^{2}\right)^{2}$.

There have been development in many numerical algorithms to solving the $\mathrm{CH}$ equation such as phase-field $[8,13,14]$, immersed boundary $[15,16]$, volume-of-fluid [17, 18], front-tracking $[19,20]$, boundary integral [21, 22], immersed interface [23, 24] and level-set [25, 26] methods. As shown in Eqs. (1.1) and (1.2), the system of equation is the fourth-order differential equation in space and it implies that there are some difficulties in numerical analysis for the $\mathrm{CH}$ equation. 
Many spatial stencils are needed and the time step restriction is stringent, for example, $\Delta t \sim h^{4}$ for using the explicit methods. Moreover, at the lower order spatial derivatives, the nonlinear terms does not guarantee numerical stability. The explicit scheme is simple but less efficient due to severe time step restriction, whereas the implicit scheme is efficient but needs large linear systems of equations to solve. Likewise, each numerical method has its own unique advantages and disadvantages for specific needs. Therefore, comparison of different schemes have been discussed to use adequate schemes for specific problems. In this paper, we focus on six widely used schemes in numerical analysis such as the the explicit Euler's, the implicit Euler's, the Crank-Nicolson, the semi-implicit Euler's, the linearly stabilized splitting and the non-linearly stabilized splitting schemes.

This paper is organized as follows. In Section 2, we describe several numerical schemes and introduce some properties related to the non-linearly stabilized splitting schemes. Numerical results are described in Section 3. Our discussion is presented in Section 4.

\section{NUMERICAL ANALYSIS}

In this section, we present fully-discrete finite-difference methods for the $\mathrm{CH}$ equation with six different schemes. We shall discretize the $\mathrm{CH}$ equation in two-dimensional domain $\Omega=$ $(a, b) \times(c, d)$. One- or three-dimensional discretizations are defined analogously. Let $N_{x}$ and $N_{y}$ be positive even integers which means a number of space step sizes in $x$ - and $y$-directions, respectively, $h=(b-a) / N_{x}=(d-c) / N_{y}$ be the uniform mesh size to both directions and the discretized domain $\Omega_{h}=\left\{\left(x_{i}, y_{j}\right): x_{i}=(i-0.5) h, y_{j}=(j-0.5) h, 1 \leq i \leq N_{x}, 1 \leq\right.$ $\left.j \leq N_{y}\right\}$ be the set of cell-centered points. Let $c_{i j}^{n}$ and $\mu_{i j}^{n}$ be approximations of $c\left(x_{i}, y_{j}, n \Delta t\right)$ and $\mu\left(x_{i}, y_{j}, n \Delta t\right)$, respectively, where $\Delta t=T / N_{t}$ is the time step, $T$ is the final time and $N_{t}$ is the total number of time steps. A vector valued phase field is defined as

$$
\mathbf{c}^{n}=\left(\begin{array}{cccc}
c_{11}^{n} & c_{12}^{n} & \cdots & c_{1 N_{y}}^{n} \\
\vdots & \vdots & \ddots & \vdots \\
c_{N_{x} 1}^{n} & c_{N_{x} 2}^{n} & \cdots & c_{N_{x} N_{y}}^{n}
\end{array}\right)
$$

We first implement the no flux boundary condition (1.3) by requiring that for each $n$,

$$
c_{0 j}^{n}=c_{1 j}^{n}, c_{N_{x}+1, j}^{n}=c_{N_{x} j}^{n}, c_{i 0}^{n}=c_{i 1}^{n}, c_{i, N_{y}+1}^{n}=c_{i N_{y}}^{n} .
$$

We define the discrete energy functional by

$$
\mathcal{E}^{h}\left(\mathbf{c}^{n}\right)=h^{2} \sum_{i=1}^{N_{x}} \sum_{j=1}^{N_{y}} F\left(c_{i j}^{n}\right)+\frac{\epsilon^{2}}{2}\left(\sum_{i=0}^{N_{x}} \sum_{j=1}^{N_{y}}\left(c_{i+1, j}^{n}-c_{i j}^{n}\right)^{2}+\sum_{i=1}^{N_{x}} \sum_{j=0}^{N_{y}}\left(c_{i, j+1}^{n}-c_{i j}^{n}\right)^{2}\right)
$$

and the discrete Laplacian by the standard five-point stencil

$$
\Delta_{d} c_{i j}=\frac{c_{i-1, j}+c_{i+1, j}-4 c_{i j}+c_{i, j-1}+c_{i, j+1}}{h^{2}} .
$$


We also define the discrete infinite norm as

$$
\|\mathbf{c}\|_{\infty}=\max _{1 \leq i \leq N_{x}, 1 \leq j \leq N_{y}}\left|c_{i j}\right| .
$$

For $M(c) \equiv 1$ which is independent of the quantity $c$ for simplicity and $F^{\prime}(c)$ which is denoted by $f(c)$, we consider several numerical schemes of Eqs. (1.1) and (1.2):

1. Explicit Euler's (EE) scheme [27]

$$
\begin{aligned}
\frac{c_{i j}^{n+1}-c_{i j}^{n}}{\Delta t} & =\Delta_{d} \mu_{i j}^{n}, \\
\mu_{i j}^{n} & =f\left(c_{i j}^{n}\right)-\epsilon^{2} \Delta_{d} c_{i j}^{n} .
\end{aligned}
$$

2. Implicit Euler's (IE) scheme [28]

$$
\begin{aligned}
\frac{c_{i j}^{n+1}-c_{i j}^{n}}{\Delta t} & =\Delta_{d} \mu_{i j}^{n+1}, \\
\mu_{i j}^{n+1} & =f\left(c_{i j}^{n+1}\right)-\epsilon^{2} \Delta_{d} c_{i j}^{n+1} .
\end{aligned}
$$

3. Crank-Nicolson (CN) scheme [5, 12, 29]

$$
\begin{aligned}
\frac{c_{i j}^{n+1}-c_{i j}^{n}}{\Delta t} & =\frac{1}{2} \Delta_{d}\left(\mu_{i j}^{n+1}+\mu_{i j}^{n}\right), \\
\mu_{i j}^{n+1} & =f\left(c_{i j}^{n+1}\right)-\epsilon^{2} \Delta_{d} c_{i j}^{n+1} .
\end{aligned}
$$

4. Semi-implicit Euler's (SIE) scheme [30, 31, 32, 33]

$$
\begin{aligned}
\frac{c_{i j}^{n+1}-c_{i j}^{n}}{\Delta t} & =\Delta_{d} \mu_{i j}^{n+1}, \\
\mu_{i j}^{n+1} & =f\left(c_{i j}^{n}\right)-\epsilon^{2} \Delta_{d} c_{i j}^{n+1} .
\end{aligned}
$$

5. Linearly stabilized splitting (LSS) scheme $[4,34]$

$$
\begin{aligned}
\frac{c_{i j}^{n+1}-c_{i j}^{n}}{\Delta t} & =\Delta_{d} \mu_{i j}^{n+1}, \\
\mu_{i j}^{n+1} & =f\left(c_{i j}^{n}\right)-2 c_{i j}^{n}+2 c_{i j}^{n+1}-\epsilon^{2} \Delta_{d} c_{i j}^{n+1} .
\end{aligned}
$$

6. Non-linearly stabilized splitting (NLSS) scheme [35, 36, 37]

$$
\begin{aligned}
\frac{c_{i j}^{n+1}-c_{i j}^{n}}{\Delta t} & =\Delta_{d} \mu_{i j}^{n+1}, \\
\mu_{i j}^{n+1} & =f\left(c_{i j}^{n+1}\right)-\epsilon^{2} \Delta_{d} c_{i j}^{n+1}+c_{i j}^{n+1}-c_{i j}^{n} .
\end{aligned}
$$

Compared with other general schemes 1-4, LSS and NLSS are known as having larger time step sizes [35]. Note that the NLSS scheme approximates the following viscous Cahn-Hilliard equation with an implicit Euler's scheme:

$$
c_{t}=\Delta\left(f(c)-\epsilon^{2} \Delta c+\nu c_{t}\right)
$$

where $\nu$ is a viscosity. To see this, let us rewrite Eq. (2.2) as 


$$
\mu_{i j}^{n+1}=f\left(c_{i j}^{n+1}\right)-\epsilon^{2} \Delta_{d} c_{i j}^{n+1}+\Delta t \frac{c_{i j}^{n+1}-c_{i j}^{n}}{\Delta t} .
$$

Therefore, the NLSS scheme approximates the viscous $\mathrm{CH}$ equation when $\nu$ equals $\Delta t$. When using a large time step, we effectively take a large viscous parameter.

In [35], Eyre proved that if $\mathbf{c}^{n+1}$ is a numerical solution of Eqs. (2.1) and (2.2) with a given $\mathbf{c}^{n}$, then

$$
\mathcal{E}^{h}\left(\mathbf{c}^{n+1}\right) \leq \mathcal{E}^{h}\left(\mathbf{c}^{n}\right)
$$

Furthermore, in [37], the authors showed the discrete energy decreasing property by using eigenvalues of the Hessian matrix of the energy functional. Using this decreasing property of the discrete total energy functional, we can show the boundedness of the numerical solution of Eqs. (2.1) and (2.2) [38]. If $\mathbf{c}^{n}$ is a numerical solution satisfying Eq. (2.4), then there exists a constant $K$, independent of $n$, such that

$$
\left\|\mathbf{c}^{n}\right\|_{\infty} \leq K
$$

Suppose Eq. (2.5) is false then there is an element $c_{i j}^{n_{K}}$ such that $\left|c_{i j}^{n_{K}}\right|>K$, where $K=$ $\sqrt{1+2 \sqrt{\mathcal{E}^{h}\left(\mathbf{c}^{0}\right) / h^{2}}}$. Since the total energy is non-increasing, we have $\mathcal{E}^{h}\left(\mathbf{c}^{0}\right)=h^{2} F(K)<$ $h^{2} F\left(\left|c_{i j}^{n_{K}}\right|\right) \leq \sum_{i=1}^{N_{x}} \sum_{j=1}^{N_{y}} h^{2} F\left(c_{i j}^{n_{K}}\right) \leq \mathcal{E}^{h}\left(\mathbf{c}^{n_{K}}\right) \leq \mathcal{E}^{h}\left(\mathbf{c}^{0}\right)$. This contradiction implies that Eq. (2.5) should be satisfied.

\section{NUMERICAL RESULTS}

In this section, we perform the following numerical experiments: finding a relation between the $\epsilon$ value and the width of the transition layer, the non-increase of the total energy, stability tests and comparison of the efficiency of CN and NLSS schemes.

3.1. The relation between the $\epsilon$ value and the width of the transition layer. In the first numerical experiment, we consider a relation between the $\epsilon$ value and the width of the transition layer for the $\mathrm{CH}$ equation. From our choice of the total energy density Eq. (1.4) and an equilibrium profile $\tanh (x /(\sqrt{2} \epsilon))$ on the infinite domain, the concentration field varies from -0.9 to 0.9 over a distance of about $2 \sqrt{2} \tanh ^{-1}(0.9)$. Therefore, if we want this value to be about $m$ grid points, the $\epsilon$ value need to be taken as follows [13]:

$$
\epsilon_{m}=\frac{h m}{2 \sqrt{2} \tanh ^{-1}(0.9)} .
$$

To confirm this, we run a simulation with the initial condition $c(x, y, 0)=0.01 \times \operatorname{rand}(x, y)$ on the domain $\Omega=(0,64) \times(0,64)$ with $h=1, \Delta t=0.1$ and $\epsilon_{4}$. Here, $\operatorname{rand}(x, y) \in[-1,1]$ is a randomly generated number. As can be observed from Fig. 2, the transition layer from $c=-0.9$ to $c=0.9$ is almost in 4 grid points regardless of type of scheme. 


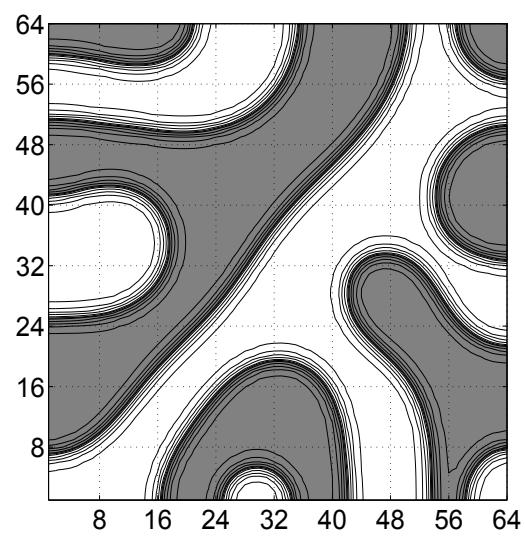

(a) $t=600$

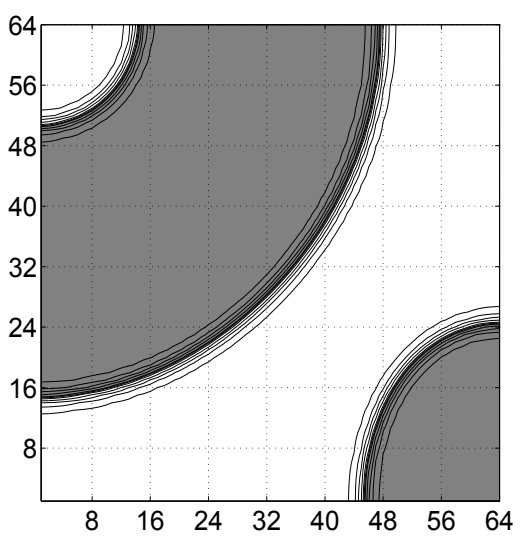

(b) $t=3000$

FIGURE 2. The contours of phase field at $c=-0.9,-0.7, \cdots, 0.9$ at time (a) $t=600$ and (b) $t=3000$.

3.2. Non-increase of the total energy. As mentioned in Section 2, NLSS inherits the energy non-increasing property. In order to numerically demonstrate the energy non-increasing property, we consider the temporal evolution of the discrete total energy. In the simulation, we choose $h=1 / 128, \Delta t=0.01$ and $\epsilon_{4}$. In Fig. 3, the temporal evolution of the non-dimensional discrete total energy $\mathcal{E}^{h}(t) / \mathcal{E}^{h}(0)$ (solid line) of the numerical solutions with the initial state $c(x, y, 0)=0.01 \times \operatorname{rand}(x, y)$. As shown in Fig. 3, the energy is non-increasing during whole time evolution. This numerical result agrees well with the total energy non-increasing property.

3.3. Stability tests. We investigate the stability of the different schemes mentioned in Section 2. We consider numerical solutions with random initial condition $c(x, 0)=\operatorname{rand}(x)$ and $c(x, y, 0)=\operatorname{rand}(x, y)$ on the unit domain for one- and two-dimensional spaces, respectively. Define $\Delta t_{c}$ be the largest time step, which satisfies the gradient stable, i.e., $\mathcal{E}^{h}\left(\mathbf{c}^{n+1}\right) \leq$ $\mathcal{E}^{h}\left(\mathbf{c}^{n}\right)$. The numerical simulations are performed on the uniform grids, $h=1 / 2^{n}$ for $n=$ $5,6,7$ and 8 . In Tables 1 and 2, we list the values of $\Delta t_{c}$ with different schemes for one- and two- dimensional spaces, respectively. From the results, we observe that EE, IE, CN and SIE schemes are not gradient stable when we use the time step larger than $\Delta t_{c}$, whereas both LSS and NLSS are unconditionally gradient stable.

Next, we also consider other numerical solutions to investigate gradient stability between LSS and NLSS with the initial data $c(x, 0)=10 \times \operatorname{rand}(x)$. For simplicity, we perform the comparison of LSS and NLSS in one-dimensional domain instead of two-dimensional one with larger random initial condition than previous simulations. In Table 3, we can observe that there is the different time step constraint in terms of numerical stability for NLSS and LSS; NLSS is still unconditionally gradient stable, but LSS is conditionally stable as shown in the reference [39]. 


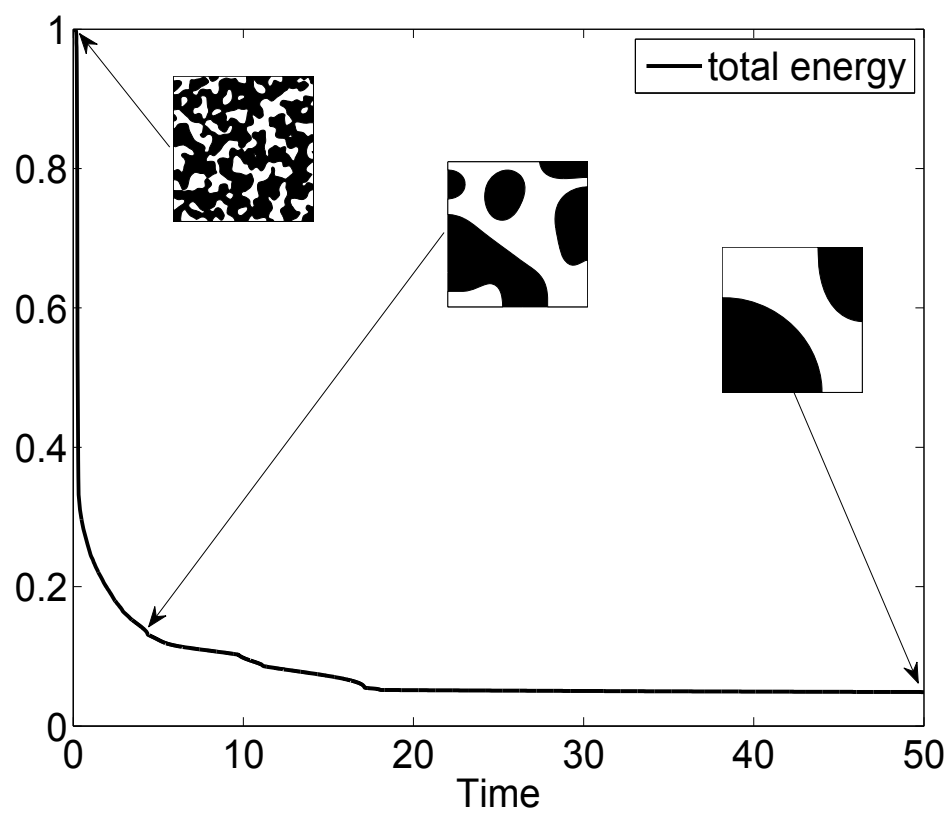

FIGURE 3. The time dependent non-dimensional discrete total energy $\mathcal{E}^{h}(t) / \mathcal{E}^{h}(0)$ (solid line) of the numerical solutions with the initial state $c(x, y, 0)=0.01 \times \operatorname{rand}(x, y)$.

TABLE 1. $\Delta t_{c}$ with different schemes for the initial condition $c(x, 0)=\operatorname{rand}(x)$.

\begin{tabular}{ccccc}
\hline Case & 32 & 64 & 128 & 256 \\
& $h=1 / 32$ & $h=1 / 64$ & $h=1 / 128$ & $h=1 / 256$ \\
\hline \hline EE & $8.0 \times 10^{-5}$ & $1.9 \times 10^{-5}$ & $4.7 \times 10^{-6}$ & $1.1 \times 10^{-6}$ \\
\hline IE & $4.9 \times 10^{-3}$ & $1.1 \times 10^{-3}$ & $2.6 \times 10^{-4}$ & $6.1 \times 10^{-5}$ \\
\hline CN & $5.3 \times 10^{-3}$ & $1.1 \times 10^{-3}$ & $1.1 \times 10^{-4}$ & $2.6 \times 10^{-5}$ \\
\hline SIE & $2.0 \times 10^{-2}$ & $2.7 \times 10^{-3}$ & $8.8 \times 10^{-4}$ & $2.2 \times 10^{-4}$ \\
\hline LSS & $\infty$ & $\infty$ & $\infty$ & $\infty$ \\
\hline NLSS & $\infty$ & $\infty$ & $\infty$ & $\infty$ \\
\hline
\end{tabular}

3.4. Comparison of the efficiency of CN and NLSS schemes. We compare the efficiency of $\mathrm{CN}$ and NLSS schemes. In the early stages of spinodal decomposition, a rapid separation of two phases occurs because the free energy $\mathcal{E}$ is high. Thus we take a small time step size in these stages. However, after the free energy has been declined sufficiently, the phases separate leisurely in the late stages. Hence we may take a large time step near the equilibrium state. In this section, we perform two tests: the first case is that the $\mathrm{CN}$ is used over whole simulations and the second case is that the $\mathrm{CN}$ scheme is used over the early stages and we alter into 
TABLE 2. $\Delta t_{c}$ with different schemes for the initial condition $c(x, y, 0)=\operatorname{rand}(x, y)$.

\begin{tabular}{ccccc}
\hline Case & $32 \times 32$ & $64 \times 64$ & $128 \times 128$ & $256 \times 256$ \\
& $h=1 / 32$ & $h=1 / 64$ & $h=1 / 128$ & $h=1 / 256$ \\
\hline \hline EE & $2.0 \times 10^{-5}$ & $5.0 \times 10^{-6}$ & $1.2 \times 10^{-6}$ & $3.2 \times 10^{-7}$ \\
\hline IE & $3.2 \times 10^{-3}$ & $8.0 \times 10^{-4}$ & $2.0 \times 10^{-4}$ & $5.0 \times 10^{-5}$ \\
\hline CN & $5.0 \times 10^{-3}$ & $1.3 \times 10^{-3}$ & $3.5 \times 10^{-4}$ & $1.0 \times 10^{-4}$ \\
\hline SIE & $9.1 \times 10^{-3}$ & $1.6 \times 10^{-3}$ & $3.5 \times 10^{-4}$ & $9.0 \times 10^{-5}$ \\
\hline LSS & $\infty$ & $\infty$ & $\infty$ & $\infty$ \\
\hline NLSS & $\infty$ & $\infty$ & $\infty$ & $\infty$ \\
\hline
\end{tabular}

TABLE 3. $\Delta t_{c}$ with different schemes for the initial condition $c(x, 0)=10 \operatorname{rand}(x)$.

\begin{tabular}{ccccc}
\hline Case & 32 & 64 & 128 & 256 \\
& $h=1 / 32$ & $h=1 / 64$ & $h=1 / 128$ & $h=1 / 256$ \\
\hline \hline LSS & $8.1 \times 10^{-6}$ & $2.0 \times 10^{-6}$ & $3.6 \times 10^{-7}$ & $8.8 \times 10^{-8}$ \\
\hline NLSS & $\infty$ & $\infty$ & $\infty$ & $\infty$ \\
\hline
\end{tabular}

the NLSS that can adopt a large time step. The reason why we use the $\mathrm{CN}$ scheme at the early stages is to acculately evolve the rapid phase separation. We decide to change the scheme when $\left\|c^{n+1}-c^{n}\right\|_{\infty}<2 \mathrm{E}-4$. In this test, $\left\|c^{n+1}-c^{n}\right\|_{\infty}$ is less than $2 \mathrm{E}-4$ within 10000 iterations. Thus, in second case, we turn on the NLSS schemes after 10000th iterations. We continue the computation until $\left\|c^{n+1}-c^{n}\right\|_{\infty}$ become less than $1 \mathrm{E}-4$. In both cases, a $256 \times 256$ mesh is used on the domain $\Omega=(0,1) \times(0,1), \epsilon_{4}$ is taken and a time step for the $\mathrm{CN}$ scheme, $\Delta t=0.00001$ and for the NLSS scheme, $\Delta t=0.0001$ was employed. Figure 4 shows the temporal evolution of each case. In the first case, we reach the $\left\|c^{n+1}-c^{n}\right\|_{\infty}<1 \mathrm{E}-4$ with 66000 iterations. In contrast, only 18000 iterations are needed to reach the same state in the second case. With less iterations, mixed scheme with the CN and the NLSS schemes can obtain the results same as those of the $\mathrm{CN}$ scheme.

\section{Conclusions}

In this paper, we investigated the performance of different schemes in terms of stability and efficiency. To compare the stability of each scheme, we performed numerical experiments such as finding the maximal time step which allows stable numerical computation with a stable initial condition. We observed that EE, IE, CN and SIE schemes are not gradient stable when we use the time step larger than the maximal time step, whereas both LSS and NLSS are unconditionally gradient stable. And, we considered evolution of numerical solution with an unstable initial condition using LSS and NLSS schemes. As a result, NLSS was still unconditionally gradient stable, but LSS was conditionally stable as shown in [39]. Finally, we compared the efficiency of CN and NLSS schemes. Owing to the advantage of NLSS which allows to use a 
(a)
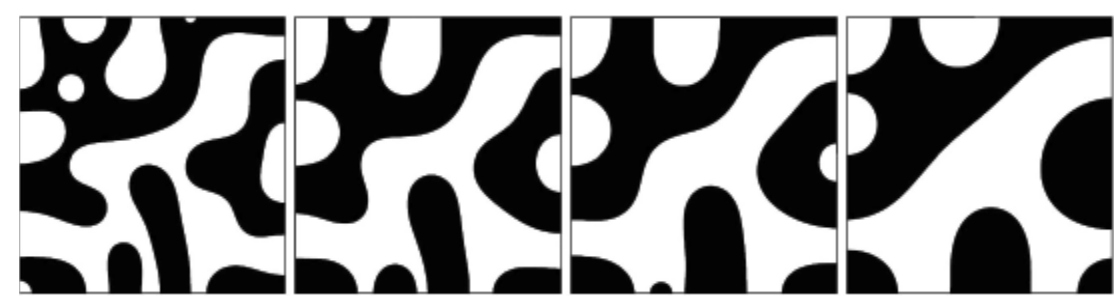

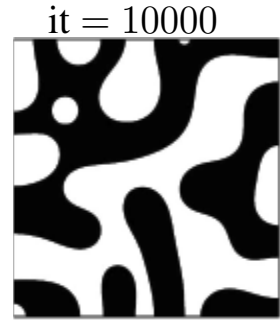

it $=10000$

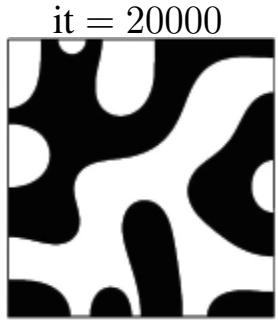

it $=12000$

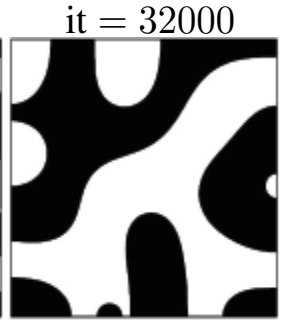

it $=14000$

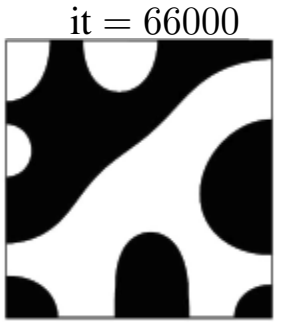

it $=18000$

FIGURE 4. The temporal evolution of morphologies during a spinodal phase separation of a binary system with different time step size (a) $\Delta t=0.00001$ using the Crank-Nicolson scheme and (b) $\Delta t=0.00001$ using the CrankNicolson scheme until 10000th iteration and $\Delta t=0.0001$ using the Nonlinearly stabilized splitting scheme after 10000th iteration, respectively. Both cases run until $\left\|c^{n+1}-c^{n}\right\|_{\infty}<1 \mathrm{E}-4$. With less iterations, mixed scheme with the CN and the NLSS schemes can obtain the results same as those of the the CN scheme.

larger time step, mixed scheme with the CN and the NLSS schemes can obtain the results same as those of the the $\mathrm{CN}$ scheme with less iterations.

\section{ACKNOWLEDGMENT}

The author (H.G. Lee) was supported by Basic Science Research Program through the National Research Foundation of Korea(NRF) funded by the Ministry of Education(20090093827).

\section{REFERENCES}

[1] J.W. Cahn, On spinodal decomposition, Acta. Metall., 9 (1961), 795-801.

[2] J.W. Cahn and J.E. Hilliard, Free energy of a non-uniform system. I. Interfacial free energy, J. Chem. Phys., 28 (1958), 258-267.

[3] J.Y. Kim, J.K. Yoon and P.R. Cha, Phase-field model of a morphological instability caused by elastic nonequilibrium, J. Korean Phys. Soc., 49 (2006), 1501-1509.

[4] A. Bertozzi, S. Esedoglu and A. Gillette, Inpainting of Binary Images Using the Cahn-Hilliard equation, IEEE Trans. Image Process., 16 (2007), 285-291.

[5] J.S. Kim, A continuous surface tension force formulation for diffuse-interface models, J. Comput. Phys., 204 (2005), 784-804. 
[6] J.S. Kim, A diffuse-interface model for axisymmetric immiscible two-phase flow. Appl. Math. Comput. 160, 589-606 (2005).

[7] J.S. Kim, K.K. Kang and J.S. Lowengrub, Conservative multigrid methods for Cahn-Hilliard fluids, J. Comput. Phys., 193 (2004), 511-543.

[8] J.S. Kim and J.S. Lowengrub, Phase field modeling and simulation of three-phase flows, Interfaces Free Bound., 7 (2005), 435-466.

[9] C.M. Elliott and D.A. French, Numerical studies of the Cahn-Hilliard equation for phase separation, IMA J. Appl. Math., 38 (1987), 97-128.

[10] H. Garcke, T. Preußer, M. Rumpf, A. Telea, U. Weikard and J. van Wijk, A phase field model for continuous clustering on vectot fields, IEEE Trans. Visual. Comput. Graph., 7 (2001), 230-241.

[11] S.M. Wise, J.S. Lowengrub, J.S. Kim, K. Thornton, P.W. Voorhees and W.C. Johnson, Quantum dot formation on a strain-patterned epitaxial thin film, Appl. Phys. Lett., 87 (2005), 133102.

[12] J.S. Kim, A numerical method for the Cahn-Hilliard equation with a variable mobility, Comm. Nonlinear Sci. Numer. Simulat., 12 (2007), 1560-1571.

[13] J.J. Eggleston, G.B. McFadden and P.W. Voorhees, A phase-field model for highly anisotropic interfacial energy, Phys. D, 150 (2001), 91-103.

[14] T. Zhang and Q. Wang, Cahn-Hilliard vs singular Cahn-Hilliard equations in phase field modeling, Commun. Comput. Phys., 7 (2010), 362-382.

[15] Q. Du and M. Li, On the stochastic immersed boundary method with an implicit interface formulation, DCDSB., 15 (2011), 373-389.

[16] J.W. Kim, D.J. Kim and H.C. Choi, An immersed-boundary finite-volume method for simulations of flow in complex geometries, J. Comput. Phys., 171 (2001), 132-150.

[17] C.R. Hirt and B.D. Nichols, Volume of fluid (VOF) method for the dynamics of free boundaries, J. Comput. Phys, 39 (1981), 201-225.

[18] S. Welch, J. Wilson, A volume of fluid based method for fluid flows with phase change, J. Comput. Phys., 160 (2000), 662-682.

[19] J. Du, B. Fix, J. Glimm, X.C. Jia, X.L. Li, Y.H. Li, Y.H. and L.L. Wu, A simple package for front tracking, J. Comput. Phys., 213 (2006), 613-628.

[20] S.O. Unverdi and G. Tryggvason, A front-tracking method for viscous, incompressible, multi-fluid flows. J. Comput. Phys., 100 (1992), 25.

[21] M. Dehghan and D. Mirzaei, A numerical method based on the boundary integral equation and dual reciprocity methods for one-dimensional Cahn-Hilliard equation, Eng. Anal. Bound. Elem., 33 (2009), 522-528.

[22] T.Y. Hou, J.S. Lowengrub and M.J. Shelley, Boundary integral methods for multicomponent fluids and multiphase materials, J. Comput. Phys., 169 (2001), 302-362.

[23] R.J. Leveque, Z. Li, The immersed interface method for elliptic equations with discontinuous coefficients and singular sources, SIAM J. Numer. Anal., 31 (1994), 1001-1025.

[24] J. Sethian and Y. Shan, Solving partial differential equations on irregular domains with moving interfaces, with applications to superconformal electrodeposition in semiconductor manufacturing, J. Comput. Phys., 227 (2008), 6411-6447.

[25] S.J. Osher and R.P. Fedkiw, Level Set Methods and Dynamic Implicit Surfaces, Springer Verlag, 2003.

[26] J.A. Sethian, Level Set Methods and Fast Marching Methods Evolving Interfaces in Computational Geometry, Fluid Mechanics, Computer Vision, and Materials Science, Cambridge University Press, 1999.

[27] F. Liu and H. Metiu, Dynamics of phase-separation of crystal-surfaces, Phys. Rev. B, 48 (1993), 5808-5817.

[28] D.J. Eyre, Systems for Cahn-Hilliard equations, SIAM J. Appl. Math., 53 (1993), 1686-1712.

[29] N. Khiari, T. Achouri, M.L. Ben Mohamed and K. Omrani, Finite difference approximate solutions for the Cahn-Hilliard equation, Numer. Methods Partial Differ. Equ., 23 (2007), 437-455.

[30] H.D. Ceniceros and A.M. Roma, A nonstiff, adaptive mesh refinement-based method for the Cahn-Hilliard equation, J. Comput. Phys., 225 (2007), 1849-1862. 
[31] M. Copetti and C.M. Elliott, Kinetics of phase decomposition processes: numerical solutions to the CahnHilliard equation, Mater. Sci. Technol., 6 (1990), 273-283.

[32] Q. Du and R. Nicolaides, Numerical studies of a continuum model of phase transition, SIAM J. Numer. Anal., 28 (1991), 1310-1322.

[33] L. Zhang, Long time behavior of difference approximations for the two-dimensional complex GinzburgLandau equation, Numer. Funct. Anal. Optim., 31 (2010), 1190-1211.

[34] R. Acar, Simulation of interface dynamics: a diffuse-interface model, Vis. Comput., 25 (2009), 101-115.

[35] D.J. Eyre, An unconditionally stable one-step scheme for gradient systems, http://www.math.utah.edu/ eyre/research/methods/stable.ps, 1998.

[36] D.J. Eyre, Unconditionally gradient stable time marching the Cahn-Hilliard equation, In: J.W. Bullard, R. Kalia, M. Stoneham and L. Chen (eds.) Comput. Math. Model. Microstructural Evolut., 1686-1712, Mater. Res. Soc., Pennsylvania, 1998.

[37] J.S. Kim and H.O. Bae, An unconditionally stable adaptive mesh refinement for Cahn-Hilliard equation, J. Korean Phys. Soc., 53 (2008), 672-679.

[38] J.S. Kim, Phase-field models for multi-component fluid flows, Communications in Computational Physics, 12 (2012), 613-661.

[39] S.M. Wise, C. Wang and J.S. Lowengrub, An energy-stable and convergent finite-difference scheme for the phase field crystal equation, SIAM J. Numer. Anal., 47(3) (2009), 2269-2288. 